\title{
Faster URLLC: Deep Learning Waveform Fingerprinting
}

\author{
Tongyang Xu and Izzat Darwazeh \\ Department of Electronic and Electrical Engineering, University College London, London, UK \\ Email: tongyang.xu.11@ucl.ac.uk, i.darwazeh@ucl.ac.uk
}

\begin{abstract}
Ultra-reliable low-latency communication (URLLC) is becoming an important research topic since it can boost mission-critical applications to serve $5 \mathrm{G}$ and its beyond. Traditionally, communication latency is determined by multiple factors such as transmission distance, signal duration and signal processing complexity. Among them, receiver side signal processing is the most critical factor. Firstly, a traditional 5G receiver will rely on independent signal processing blocks to decode a message, which is not optimal and would introduce unnecessary processing delay. Secondly, unsuccessful signal decoding will trigger retransmission and extra latency will be caused. This work proposes a waveform fingerprinting (WF) framework, which can easily identify users and resource assignment information based on signal patterns. More importantly, the WF scheme removes the block based 5G signal processing mechanism leading to a faster signal identification. A deep learning based intelligent identifier is integrated to assist the faster signal identification. Simulation results reveal that the proposed WF scheme enables higher reliability than the traditional 5G block based processing especially when noise power is higher than signal power. In addition, the processing latency of the WF scheme is two orders of magnitude shorter than the traditional $5 \mathrm{G}$ block based scheme.

Index Terms-URLLC, waveform, fingerprinting, deep learning, signal classification, SEFDM, non-orthogonal.
\end{abstract}

\section{INTRODUCTION}

In 5G, ultra-reliable low-latency communication (URLLC) is a critical technique for time-sensitive applications [1] such as autonomous driving, industry 4.0, eHealth and financial services. However, the design of URLLC might be the most challenging [2] since it has to meet both ultra-reliable and low-latency, which are mutually contradictory requirements.

The time and frequency resources allocated to a user equipment (UE) is decided by downlink control information (DCI) in physical downlink control channel (PDCCH) [3]. To let a UE accurately identify the correct resource assignment, the network typically allocates a unique radio network temporary identifier (RNTI) to each UE when the UE initially registers to the network. To avoid extra controlling overhead, the RNTI is not sent as an independent message but is used to mask cyclic redundancy check (CRC) messages in 5G. The mask operation is regarded as a bit-by-bit scrambling operation on the CRC message using the RNTI sequence. To correctly recover the control information from DCI, a UE will use its RNTI to blindly decode a set of pre-defined candidate CRC packets. Once the CRC is unmasked and passes CRC check, the correct DCI messages will be obtained with the resource assignment information. However, the failure of CRC check could happen due to incorrect RNTI blind CRC decoding or unexpected signal transmission errors. In this case, the receiver will discard the data and ask for re-transmission. Therefore, the uncertainty of traditional DCI decoding could cause longer and random processing latency at the receiver.

The aim of this work is to simplify the receiver side DCI decoding by allocating intelligence to each UE device. A successful communication is achieved on the basis of accurate unmasking of CRC and the successful CRC check. One step failure will result in incorrect resource assignment information extraction and unexpected re-transmission. To avoid the aforementioned error-sensitive signal processing, this work applies an intelligent UE side processing strategy for user resource assignment identification using intelligent waveform fingerprinting. The basic idea is to apply a non-orthogonal signal waveform termed spectrally efficient frequency division multiplexing (SEFDM) [4], which can adaptively compress spectral bandwidth while maintaining the same data rate. The spectral compression, defined by bandwidth compression factor (BCF), could be used as a unique label for user identity. To accurately identify each signal pattern, a deep learning framework will be used in this work. Therefore, an intelligent waveform fingerprinting framework will be designed in this work to simplify the traditional DCI decoding and will lead to faster URLLC.

\section{LATENCY MODEL IN 5G}

In $4 \mathrm{G}$ long term evolution (LTE), a basic time resource assignment is fixed to a duration of $1 \mathrm{~ms}$, which represents one transmission time interval (TTI) or one subframe. However, $5 \mathrm{G}$ is more flexible and a shorter TTI, noted as a mini-slot [5], is applicable. A conventional time slot includes 14 orthogonal frequency division multiplexing (OFDM) symbols while the mini-slot could have 2, 4, or 7 OFDM symbols. In addition, due to the flexible numerology design for $5 \mathrm{G}$, the sub-carrier spacing is not limited to the $15 \mathrm{kHz}$ in $4 \mathrm{G}$. There are more options such as $30 \mathrm{kHz}, 60 \mathrm{kHz}$ and $120 \mathrm{kHz}$. In this case, one TTI in 5G, including 14 OFDM symbols, ranges from $0.125 \mathrm{~ms}$ to $1 \mathrm{~ms}$. Therefore, to have a low-latency real-time communication, the overall signal processing time should be less than $0.125 \mathrm{~ms}$.

End-to-end latency is commonly investigated, which is the one-way signal transmission duration from a base station (BS) to a UE. However, practical communication systems have transmission errors and re-transmission of a signal is 


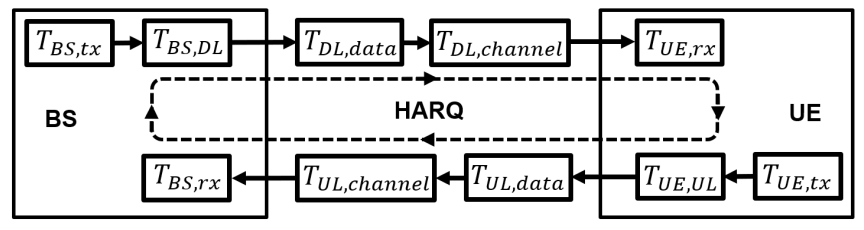

Fig. 1. Round-trip latency model for 5G.

unavoidable. Therefore, round-trip latency is more realistic for practical system latency evaluation. The theory behind a round-trip communication is based on hybrid automatic repeat request (HARQ) [6]. When a receiver decodes successfully a message via passing CRC check, an acknowledgement (ACK) will be sent back to a base station. Otherwise, a negativeacknowledgement (NACK) will be sent to the base station for re-transmission.

A general round-trip communication framework [3], [7] is illustrated in Fig. 1. $T_{B S, t x}$ indicates the time duration required to process data from higher communication layers (HCL) and lower communication layers (LCL). $T_{B S, D L}$ represents the queueing time for a frame integrating in an available $5 \mathrm{G}$ downlink (DL) slot. $T_{D L \text {,data }}$ represents the data transmission duration, which is decided by the number of OFDM symbols and sub-carrier spacing. A smaller number of OFDM symbols will have a shorter $T_{D L \text {,data }}$ and thus low latency. In addition, a larger sub-carrier spacing will lead to a shorter time duration for one OFDM symbol. $T_{D L \text {,channel }}$ indicates the time duration of a signal transmitted over the air. The longer transmission distance between a base station and a UE will cause a longer latency. $T_{U E, r x}$ is the processing time duration between signal reception and the end of signal decoding. This stage contributes a lot to the entire communication latency since signal compensation, demodulation and decoding are all within this stage. The processing of this stage is very important since the decoding result will determine whether a re-transmission is needed or not. $T_{U E, t x}$ is the duration for ACK/NACK generation. $T_{U E, U L}$ is the queueing time for the ACK/NACK frame integrating in an available $5 \mathrm{G}$ uplink (UL) slot. $T_{U L, \text { data }}$ is the time interval of the UL frame, which could occupy a few number of OFDM symbols period. $T_{U L, \text { channel }}$ indicates the time duration of the UL ACK/NACK packet transmitted over the air. $T_{B S, r x}$ indicates the time interval to decode the ACK/NACK packet.

Therefore, the overall round-trip latency [3] for a communication is computed as

$$
T_{\text {total }}=T_{1}+m \times T_{H A R Q},
$$

where $m$ indicates the number of re-transmission. Each term in (1) is defined as

$$
\begin{aligned}
& T_{1}=T_{B S, t x}+T_{B S, D L}+T_{D L, \text { data }}+T_{D L, \text { channel }}+T_{U E, r x} \\
& T_{2}=T_{U E, t x}+T_{U E, U L}+T_{U L, \text { data }}+T_{U L, \text { channel }}+T_{B S, r x} \\
& T_{H A R Q}=T_{1}+T_{2} .
\end{aligned}
$$

As mentioned by [1], over-the-air transmission time durations $T_{D L, \text { channel }}$ and $T_{U L \text {,channel }}$ have limited impacts on the entire end-to-end latency. The time duration $T_{D L, \text { data }}$, $T_{U L, \text { data }}$ for each frame has been optimized from $1 \mathrm{~ms}$ to $0.125 \mathrm{~ms}$, which is the key improvement from $4 \mathrm{G}$ to $5 \mathrm{G}$ in terms of URLLC. However, the time delays from queueing $T_{B S, D L}, T_{U E, U L}$ and UE signal processing $T_{U E, r x}$ are key bottlenecks to further speed up URLLC.

Among the limitation factors, the signal processing time duration $T_{U E, r x}$ at a UE is the key factor causing longer latency since a unsuccessful message decoding will cause retransmission as mathematically described in (1). Therefore, to quickly and accurately identify users and extract resource assignment information from PDCCH packets is of great importance and will be the motivation of this work.

\section{WAVEFORM FINGERPRINTING FOR FASTER URLLC}

To identify assigned resource blocks for a unique user, the DCI has to be extracted first using the UE RNTI number. One way to simplify this process is to modify signal characteristics for assigned resources with unique and distinguishable features. This work employs the non-orthogonal signal waveform, SEFDM [4], which maintains the same data rate (i.e. same frame duration) compared to 5G OFDM, but compresses subcarrier spacing. In this case, the occupied spectral bandwidth will be adaptive based on the sub-carrier spacing compression ratio. Each compression ratio will be used as an identifier for a specific user and the associated signal band will be identified and allocated to that user. This automatic signal identification will simplify the UE side signal processing and the identification process is robust to transmission errors. It indicates that even the transmission has errors and cannot pass CRC check, the user and resource identification still works. This can avoid redundant signal re-transmission when a signal cannot pass CRC check due to transmission errors or failed RNTI unmasking.

\section{A. Principle of Waveform}

The basic principle of the non-orthogonal waveform is shown in Fig. 2. It should be noted that each impulse represents a sub-carrier and all the cases in Fig. 2 have the same spectral bandwidth per sub-carrier. The sub-carrier spacing for OFDM in Fig. 2(a) is labelled as $\Delta f$. Due to the spectral compression advantage, the sub-carrier spacing for SEFDM in Fig. 2(b), denoted as $\Delta f_{1}$, is smaller than $\Delta f$. The bandwidth compression factor, termed $\alpha_{1}$, is allocated to the SEFDM signal in Fig. 2(b). The value of $\alpha_{1}$ is fractional and $\alpha_{1}<1$. Further spectral bandwidth compressions can be achieved in Fig. 2(c)(d) with configurations as $\Delta f_{3}<\Delta f_{2}<\Delta f_{1}<\Delta f$ and $\alpha_{3}<\alpha_{2}<\alpha_{1}$. Based on the examples in Fig. 2, four users could be supported via using the four different signals. The mathematical expression for a SEFDM signal is defined as

$$
X_{k}=\frac{1}{\sqrt{Q}} \sum_{n=0}^{N-1} s_{n} \exp \left(\frac{j 2 \pi n k \alpha}{Q}\right),
$$




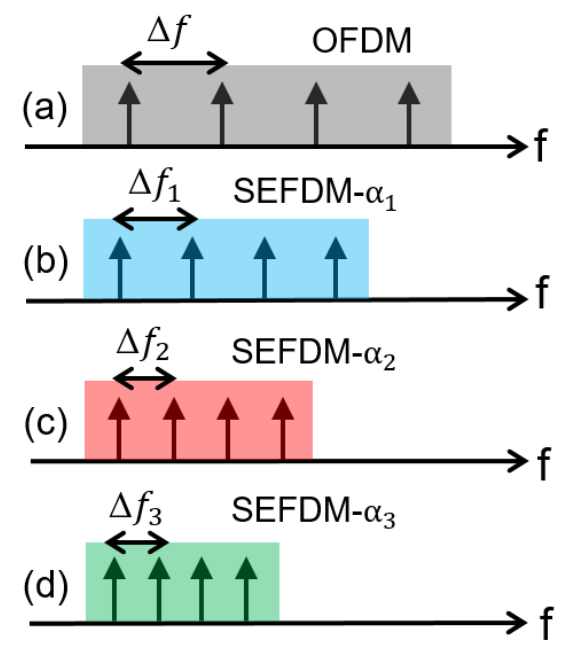

Fig. 2. Principle of SEFDM signal waveform and the illustration for its feasibility as user identity. Each impulse represents a sub-carrier and $(a)(b)(c)(d)$ have the same bandwidth per sub-carrier but with different sub-carrier spacing $\Delta f_{3}<\Delta f_{2}<\Delta f_{1}<\Delta f$. In this example, (a)(b)(c)(d) can represent four different users.

where the parameters are defined as

- $X_{k}$, the time sample with the index of $k=0,1, \ldots, Q-1$.

- $Q=\rho N$, the number of time samples.

- $N$, the number of sub-carriers.

- $\rho$, the oversampling factor.

- $\frac{1}{\sqrt{Q}}$, the scaling factor.

- $s_{n}$, the $n^{\text {th }}$ single-carrier symbol in one SEFDM symbol.

- $\alpha=\Delta f \cdot T$, the bandwidth compression factor where $\Delta f$ is the sub-carrier spacing and $T$ is the time duration of one SEFDM symbol.

\section{B. Traditional 5G PDCCH Decoding}

The user identity information RNTI and its corresponding resource assignment details are included in PDCCH. A general PDCCH signal generation and reception architecture is presented in Fig. 3.

At the transmitter, the control information is encoded by a CRC encoder, which will add CRC redundancy bits at the end. To guarantee that the control message can be sent to a specific UE, the user identity number RNTI should be included. The efficient way to add the RNTI number is to use it to scramble the CRC redundancy bits. In this case, only the right UE, with the right RNTI number, can descramble the message and pass the CRC check. After the scrambling, Polar code is used to encode the control message to mitigate potential wireless channel effects. To achieve a specific coding rate, rate matching is applied. The binary bit sequence is then QPSK mapped to obtain complex symbols. At the end, the OFDM modulation block is applied to modulate each symbol on allocated sub-carriers. The multi-carrier OFDM signal will be transmitted over the air with distortions from frequency selective channels and additive white Gaussian noise (AWGN).

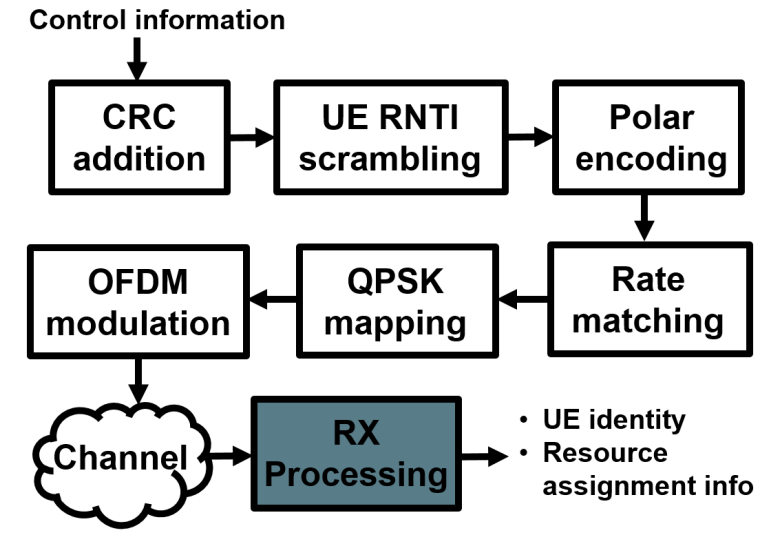

Fig. 3. Block diagram of PDCCH signal generation and reception.

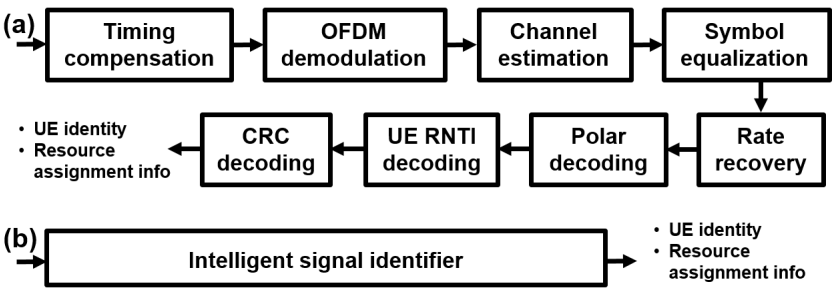

Fig. 4. Receiver side signal processing for user identity and resource assignment information. (a) Traditional PDCCH decoding. (b) Proposed intelligent signal identifier based on waveform fingerprinting.

At the traditional 5G receiver, as illustrated in Fig. 4(a), timing offset has to be compensated before an accurate message decoding. The compensated multi-carrier signal will go through OFDM demodulation and output single-carrier symbols. Channel estimation is operated in frequency-domain and the estimated channel coefficients are used to equalize the single-carrier symbols using a one-tap equalizer. The equalized PDCCH symbols will go through rate recovery before Polar decoding. Then the descrambling is operated using UE's RNTI number. The CRC redundancy bits of a target PDCCH packet should be correctly unmasked using the UE's RNTI. If the unmasked message can pass CRC check, the exact resource assignment details will be extracted.

However, the receiver side process in the traditional $5 \mathrm{G}$ system has the following drawbacks:

- RNTI error. Since a UE has no way to immediately identify the message that is sent to him, therefore the UE will blindly attempt a set of PDCCH packets and using its RNTI to find the right packet. It is inferred that this blind decoding delay is not deterministic. The best case is that the first attempt is successful while the worst case is no solution in the set of candidates.

- Transmission error. It is difficult to judge whether the CRC check failure comes from transmission errors or incorrect RTNI attempts. The failure of CRC check could be caused by transmission errors in non-stationary wireless environments even using a correct UE's RNTI 


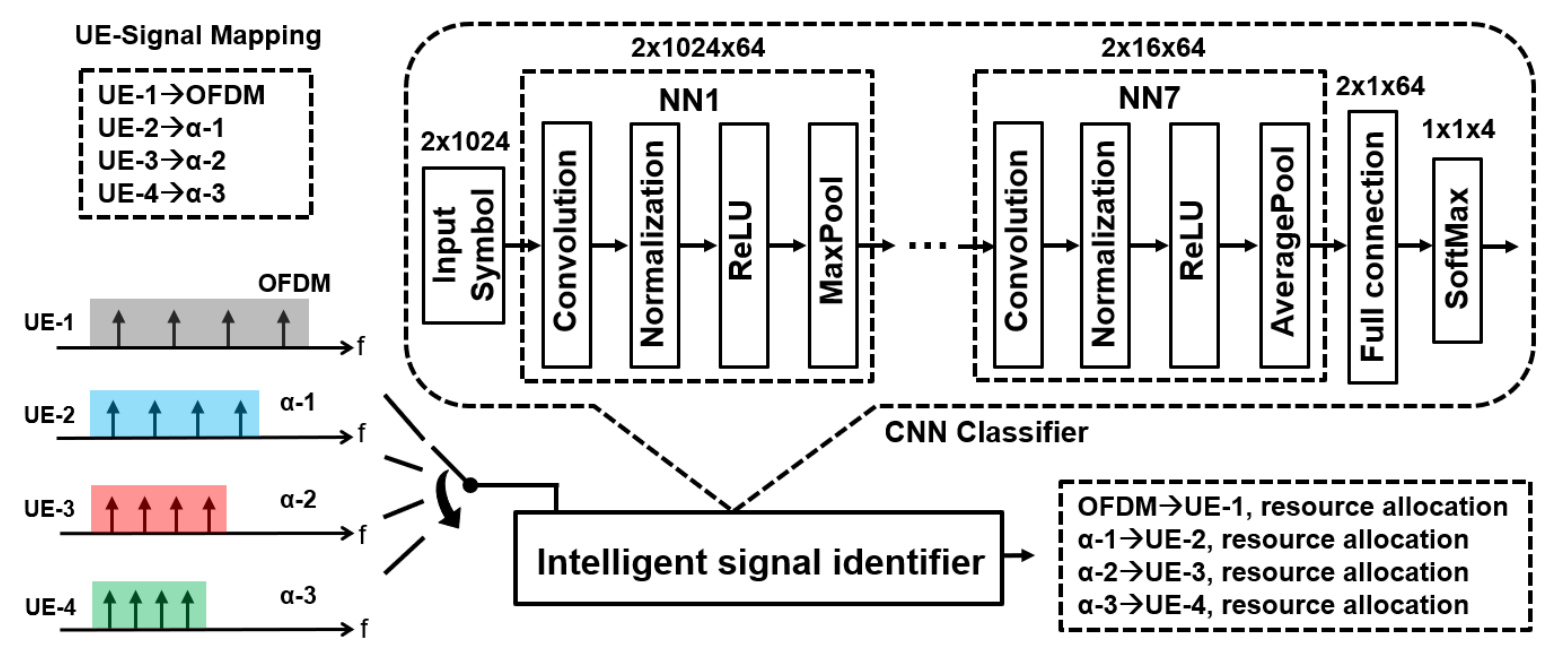

Fig. 5. Deep learning CNN signal identifier architecture.

number. In this case, the UE could discard the message that belongs to it and check other candidate packets. This will cause additional and random time delay. The worst case is that all the attempts are unsuccessful either due to transmission errors or incorrect RNTI unmasking. Retransmission will be requested and will cause round-trip time delay.

- Signal processing error. The block based receiver architecture cannot guarantee reliable signal processing. Timing compensation, channel estimation and channel equalization might not be perfect. This will cause signal processing errors at the receiver, which will cause the failure of CRC check as well.

- Coding redundancy. The traditional downlink control message decoding requires Polar coding to guarantee the correct decoding of DCI information. Therefore, extra coding redundancy will be required leading to reduced spectral efficiency.

To reduce the re-transmission request and cut signal processing delay at a UE, intelligent solutions are possible such as the deep learning identifier shown in Fig. 4(b), which can automatically learn signal features and identify raw signals from waveform fingerprints before any receiver side signal processing.

\section{Proposed Intelligent Signal Identifier}

A specific UE-signal mapping scheme is pre-defined as shown in Fig. 5. Each UE is uniquely correlated with a signal pattern. An intelligent signal identifier will identify the value of $\alpha$ for each incoming signal. Then the $\alpha$ labelled UE will be identified with its allocated resources.

Intelligent signal identification has been successfully applied and implemented for single-carrier modulation classification [8] and later applied in multi-carrier signal classification [9]. The commonly used intelligent solution for signal classification is deep learning since it can automatically extract signal hidden features, in which unique features are applied to identify each signal. One representative deep learning method is convolutional neural network ( $\mathrm{CNN})$, which employs multiple convolutional layers for automatic feature extraction and its feature extraction principle is mathematically explained below

$$
\begin{aligned}
& L_{0}=f\left(Y ; \phi_{0}, \phi_{1}, \ldots, \phi_{K-1}\right) \\
& L_{1}=f\left(L_{0} ; \omega_{0}, \omega_{1}, \ldots, \omega_{K-1}\right) \\
& \vdots \\
& L_{\Omega-1}=f\left(L_{\Omega-2} ; \sigma_{0}, \sigma_{1}, \ldots, \sigma_{K-1}\right),
\end{aligned}
$$

where $L_{0}$ indicates the feature maps after the 1 st convolutional layer operations, $f(\cdot)$ indicates a convolution operation, $Y$ is the received signal immediately after the channel, $\left(\phi_{0}, \phi_{1}, \ldots, \phi_{K-1}\right)$ represents $K$ feature filters at the 1 st convolutional layer. There will be $K$ convolution operations between $Y$ and $\phi$. Therefore, $L_{0}$ includes $K$ feature maps. The similar operations will be repeated for the following convolutional layers until all the $\Omega$ convolutional layers are went through. The final feature maps are noted as $L_{\Omega-1}$, which will be used by a classification function as below

$$
C_{c n n}=\xi\left(L_{\Omega-1} ; W\right),
$$

where $\xi(\cdot)$ represents classification function and $W$ indicates full connection functions.

It is noted that the input to the CNN feature learning process (4) is the original signal $Y$, which is not pre-processed by any compensation algorithms. The CNN will automatically learn hidden features that are robust to channel and hardware impairments.

The specific CNN classifier architecture designed for this work is demonstrated in Fig. 5 where multiple neural network (NN) sub-blocks are packed to realize a deep neural network structure. This work will configure multi-carrier signals with 1024 sub-carriers. Taking into account QPSK symbols, real 
and imaginary part of a symbol will lead to a $2 \times 1024$ input symbol matrix.

For the first six NN sub-blocks, four sub-layers are included, namely convolutional layer, normalization layer, ReLU layer and MaxPool layer. Each layer has its unique function. The convolutional layer works as a filter. This work designs $K=64$ feature filers and after the convolutional layer there will be 64 independent feature maps. Therefore, the dimension of the first NN sub-block becomes $2 \times 1024 \times 64$. Each feature map will contain useful hidden feature information. The convolutional layer output will be normalized and then fed to the ReLU activation function. The MaxPool layer is used to down sample the ReLU layer output and reserve extreme features. The dimension of each NN sub-block will be decreased and it will be simplified into $2 \times 16 \times 64$ at the last NN sub-block. The same sub-layer structure will be followed for other NN sub-blocks except the last NN subblock, in which an AveragePool layer is applied instead of the MaxPool layer. The reason is to get smooth features here instead of extreme features. Once the hidden feature is extracted, a full connection layer and a SoftMax layer will work together for signal classification. The cross-entropy loss between predicted signal classes and true signal classes will be minimized by the stochastic gradient descent with momentum (SGDM) optimizer. After pre-defined training iterations with backpropagation operations, the optimal CNN classifier will be obtained.

\section{Simulation Setup and Results}

\section{A. Simulation Environment}

To have a convincing simulation result, this work follows the 5G downlink control processing model in Matlab [10]. The framework applies a blind RNTI search decoding mechanism since a UE has no information of the control channel architecture. It will pre-define a set of PDCCH search candidates and a UE will use its own RNTI to find and decode the right candidate. Once the decoded message passes CRC check with returned zero checksum, the UE successfully finds its control information packet and assigned resources.

In this simulation, PDCCH aggregation level is configured to be one, which indicates the number of control channel element (CCE) is one. According to the 5G DL framework [10], there are four possible sub-carrier mapping schemes at the first aggregation level. Here we consider the forth option and the total number of available bits will be 108 bits. The inverse fast Fourier transform (IFFT) size is 1024 and an OFDM signal will have 1024 time samples. This simulation follows the TDL-A channel model with a $30 \mathrm{~ns}$ delay spread profile. In addition, this work considers a single-antenna communication scenario. For the traditional 5G downlink control channel, to decode Polar coded messages, a successive-cancellation list decoder is applied at the receiver with the length of decoding list of 8. At UE side, the receiver has no information of the sub-carrier packing scheme. Therefore, a set of search candidates are pre-defined. In this simulation, we consider

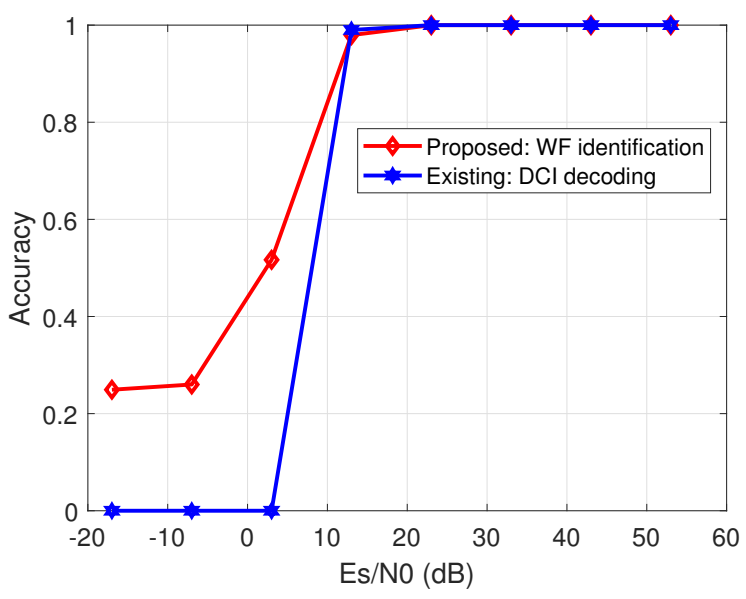

Fig. 6. Accuracy comparison for reliability evaluation.

a small candidate set with aggregation levels of 1, 2, 4, with the number of candidates being 4, 2, 1, respectively. Fundamentally, the UE will need to search all the candidates per aggregation level and find the correct one with zero CRC result. However, the search could be early terminated when the right solution is found during the search. This is the reason why the blind search causes extra and random time latency.

\section{B. Training Dataset Collection}

To have a fair comparison with the traditional $5 \mathrm{G}$ receiver DCI decoding, the CNN training dataset will follow the $5 \mathrm{G}$ signal standard. As shown in Fig. 3, either the traditional 5G DCI decoding or the proposed intelligent signal classifier will be within the 'RX Processing' block. Therefore, both schemes follow the 5G signal format. The intelligent $\mathrm{CNN}$ classifier has to collect training data first and train a working model offline. Once a model is obtained, there will be no additional training anymore and the model can be reused for future purposes.

At the training stage, 1024 time samples will be captured for each received symbol even without perfect timing synchronization. As noted that the fourth sub-carrier packing scheme at the first PDCCH aggregation level is considered, therefore this work will consider four signal patterns with the following configurations: OFDM, SEFDM $(\alpha=0.9)$, SEFDM $(\alpha=0.8)$, SEFDM $(\alpha=0.7)$. The training stage will collect 2,000 symbols for each signal class and there will be overall 8,000 SEFDM/OFDM symbols for training a CNN classifier.

\section{Simulation Results}

1) Reliability: Unlike the traditional bit error rate (BER) metric measurement for reliability, this work will use identification accuracy as the metric. Accuracy for traditional 5G DCI decoding indicates the probability of successful DCI decoding with zero CRC checksum. On the other hand, accuracy for our proposed waveform fingerprinting scheme is the ratio of correctly recognized symbols to total evaluated symbols.

In Fig. 6, it is clear that both schemes can achieve $100 \%$ accuracy when signal power is large sufficient. The accuracy will 


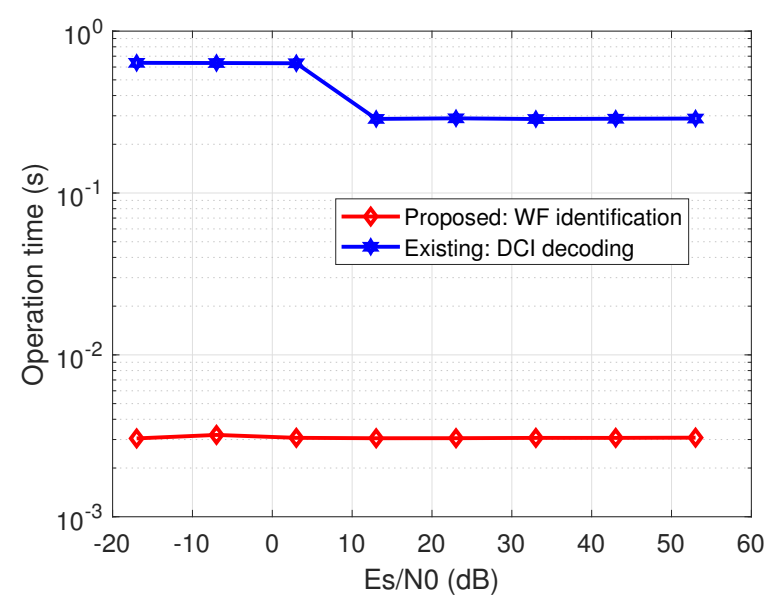

Fig. 7. Operation time comparison for latency evaluation.

drop significantly when noise power dominates especially for Es/N0 below zero. In the low Es/N0 regime, the WF scheme still has chances to successfully decode messages while the traditional DCI decoding will always fail. It reveals that the WF scheme has higher reliability than the traditional DCI decoding especially when noise power dominates. A higher accuracy rate indicates a lower probability of re-transmission, which will therefore shorten communication latency.

2) Latency: This work measures processing latency in Matlab using an off-the-shelf CPU (Intel(R) Core(TM) i7$6700 \mathrm{CPU} @ 3.4 \mathrm{GHz}$ ). For the traditional UE processing, a number of operations are needed as shown in Fig. 4(a). For our proposed waveform fingerprinting framework, a single processing block, intelligent signal identifier, is required in Fig. 4(b). Fig. 7 demonstrates that the traditional DCI decoding scheme has a longer latency than the proposed WF scheme. This is expected since multiple signal processing modules are needed for DCI decoding in Fig. 4(a) while the WF scheme in Fig. 4(b) only needs one processing module. In addition, a UE has to go through a set of pre-defined PDCCH candidates and find the one that can pass the CRC check. However, the WF framework will automatically evaluate the received signal features and classify it to a proper user. Moreover, an important discovery is the dynamic operation latency by the DCI decoding. In Fig. 7, it is obvious that the DCI decoding requires longer time at low Es/N0 regime. This is due to the high noise power such that all the candidates have to be searched. When signal power is increased, the CRC check will be successful at the correct candidate and early termination will avoid redundant search.

This work applies hardware CPU to measure operation latency, which will not be scientifically accurate compared to $5 \mathrm{G}$ transceiver hardware. To have a fair comparison for the two schemes using CPU, this work ensures the same simulation operation environment for both cases. Based on the time profile from Matlab, the latency for the traditional DCI decoding is roughly $0.6 \mathrm{~s}$ at low Es/N0 regime and $0.3 \mathrm{~s}$ at high Es/N0 regime while the latency is reduced to $0.003 \mathrm{~s}$ for the WF scheme. The WF latency is more than two orders of magnitude lower than the DCI decoding latency.

\section{CONClusion}

This work proposed a new $5 \mathrm{G}$ receiver side control channel processing scheme, termed waveform fingerprinting (WF), which can enable faster URLLC. It can assist a UE to identify the right PDCCH packet and assign resources in a faster and simpler way. A non-orthogonal signal waveform is integrated in a $5 \mathrm{G}$ framework and the waveform unique pattern can be used as the identifier for each UE. By adaptively changing spectral characteristics, multiple non-orthogonal waveform patterns are obtained and therefore multiple UEs can be supported based on the dynamic waveform tuning. Simulation is designed based on an available 5G framework. Firstly, results reveal that the WF scheme achieves higher reliability than the traditional $5 \mathrm{G}$ receiver signal processing especially when signal is greatly distorted by noise. Secondly, results show that the WF scheme will use a shorter time to process incoming packets. The processing latency of the WF scheme is more than two orders of magnitude shorter than the traditional $5 \mathrm{G}$ signal processing.

\section{ACKNOWLEDGEMENT}

This work was funded by the Engineering and Physical Sciences Research Council (EPSRC) grant (EP/P022723/1) for the research on "The Internet of Silicon Retinas (IoSiRe): Machine to machine communications for neuromorphic vision sensing data". This work was also funded by EPSRC Grant (EP/S028455/1) on "Learning to Communicate (LeanCom): Deep Learning based solutions for the Physical Layer of Machine Type Communications".

\section{REFERENCES}

[1] C. She, C. Sun, Z. Gu, Y. Li, C. Yang, H. V. Poor, and B. Vucetic, "A tutorial on ultra-reliable and low-latency communications in 6G: Integrating domain knowledge into deep learning," arXiv e-prints, p. arXiv:2009.06010, Sep. 2020.

[2] H. Ji, S. Park, J. Yeo, Y. Kim, J. Lee, and B. Shim, "Ultra-reliable and low-latency communications in 5G downlink: Physical layer aspects," IEEE Wireless Communications, vol. 25, no. 3, pp. 124-130, 2018.

[3] Rohde\&Schwarz, 5G New Radio: Fundamentals, procedures, testing aspects. Rohde\&Schwarz, 2020.

[4] T. Xu and I. Darwazeh, "Transmission experiment of bandwidth compressed carrier aggregation in a realistic fading channel," IEEE Transactions on Vehicular Technology, vol. 66, no. 5, pp. 4087-4097, May 2017.

[5] E. Dahlman, S. Parkvall, and J. Sköld, 5G NR: The Next Generation Wireless Access Technology. Academic Press, 2018.

[6] 3GPP TS 38.214 version 15.3.0, "5G NR; physical layer procedures for data," Rel. 15, Oct. 2018.

[7] 3GPP TS 22.104 version 16.5.0, "5G; service requirements for cyberphysical control applications in vertical domains," Rel. 16, Sept. 2020.

[8] T. J. O'Shea, T. Roy, and T. C. Clancy, "Over-the-air deep learning based radio signal classification," IEEE Journal of Selected Topics in Signal Processing, vol. 12, no. 1, pp. 168-179, Feb. 2018.

[9] T. Xu and I. Darwazeh, "Deep learning for over-the-air non-orthogonal signal classification," in 2020 IEEE 91st Vehicular Technology Conference (VTC2020-Spring), 2020, pp. 1-5.

[10] MathWorks, "Downlink Control Processing and Procedures," https://uk.mathworks.com/help/5g/ug/downlink-control-processingand-procedures.html, Dec 2020. 